\title{
Modelling the spatial spread risk of plant pests and pathogens for strategic management decisions
}

\author{
$\underline{\text { P.W.J. Baxter }}^{\text {a,b }}$, A. Woodley ${ }^{\text {c }}$ and G. Hamilton ${ }^{\text {a,b }}$ \\ a Plant Biosecurity Cooperative Research Centre, Bruce, Australian Capital Territory, Australia \\ ${ }^{b}$ School of Earth, Environmental and Biological Sciences, Queensland University of Technology (QUT), \\ Brisbane, Queensland, Australia \\ ${ }^{c}$ School of Electrical Engineering and Computer Science, Queensland University of Technology (QUT) and \\ Institute for Future Environments, Brisbane, Queensland, Australia \\ Email:p3.baxter@qut.edu.au
}

\begin{abstract}
Invasive pests and pathogens pose a grave and growing threat to agricultural systems, threatening food security and access to domestic and export trade, as well as having potentially serious environmental and social impacts. Management responses to pest or pathogen incursions include planning for conducting surveillance, implementing quarantine restrictions and treating infected farms. Smart and transparent decisions need to be made to control the spread of an incursion, often in the context of sparse information. Given the array of management options, modelling tools can help greatly in more targeted spatial prioritisation of surveillance and quarantine as the incursion response unfolds.
\end{abstract}

We constructed a simulation model to allow managers of incursions to explore the possible effects of their decisions. The model operates at the farm-level of spatial resolution, with risk pathways parameterised via flexible networks of connections between farms. It models growth of the pest organism within farms, its spread between farms by natural or human-assisted means, and its detection and control by land-owners or managers. Quarantine options within the model can range from isolation of an individual property to regionwide restrictions. Surveillance options range from straightforward ranking by estimated risk to more sophisticated search prioritisation algorithms. The model continually updates risk estimates from previous surveillance results and ongoing prevailing risk propagated through the connection pathways. Initial risk estimates can incorporate tracing data, wherein response managers have documented previous high-risk pathways connected to properties with known infections. Simulated surveillance results can be replaced by actual surveillance history data, if available.

Users such as biosecurity managers can explore model features and scenarios through the interactive model visualisation software "BIOSPARK". Whereas the model is underpinned by simulation of within-farm growth and between-farm spread of the invading organism, the output for response program managers is primarily presented as visualised expected risk to farms of unknown pest status, and their ranked risk levels, to aid in guiding surveillance decisions. Risk is visualised as heat maps overlaid on the landscape, and can be viewed over each time step of the analysis. Sites recommended for surveillance are presented as a ranked list, with their locations flagged on the landscape.

We have retained sufficient generality in the model, so that future developments can include considerations of different organisms, flexibility in number of risk networks to be entered, and application of the model to different jurisdictions. There is a need for model-based tools such as this to aid in framing, supporting and communicating agricultural management decisions in complex and challenging situations.

Keywords: Biosecurity, invasive species, threatening processes, prioritisation, simulation 


\section{INTRODUCTION}

Invasive species can have severe impacts on agricultural productivity, food security, and the economy and environment in general (Pimentel et al. 2000), and the number of incursions is set to continue increasing due to global trade (Westphal et al. 2008). Invasive fungal pathogens can inflict similarly serious economic costs on agriculture through both direct losses of yield, and interrupted productivity during control operations (Murray and Brennan 2010; Cook et al. 2013). For example a recent incursion of Panama Disease Tropical Race 4 in northern Queensland, caused by the pathogen Fusarium oxysporum f. sp. cubense, has been projected to cost the banana industry in the region of $\$ 138 \mathrm{~m}$ annually (Cook et al. 2015).

There is a compelling need to control and limit the spread of incursions quickly after detection (Simberloff 2003), as early intervention can avoid disproportionately large costs into the future. However many threatening organisms are difficult to detect and control, and search strategies can be hampered by a lack of data and knowledge about their potential spread (Baxter and Possingham 2011). In the face of such uncertainty, considerable system variability, and limited management resources, interactive decision tools can provide an essential means of understanding likely spread pathways under different scenarios, and formulating a strategic response to incursions. While various models for estimating spread of invasive species exist (e.g. Rasmussen \& Hamilton 2012), these are not typically designed to include the risk pathways of agricultural landscapes. Visual decision tools in particular can also serve the purpose of public communication, which is essential for ongoing public support of biosecurity operations (Barkley et al. 2014).

Here we present a model of the introduction, spread, detection and control of a generic fungal pathogen. The model underpins an interactive visualisation platform ("BIOSPARK") that can serve as a decision support tool for biosecurity managers in the context of new or ongoing responses to incursions of threatening organisms. The model simulates system dynamics allowing for a range of risk pathways and management options, allows for user-controlled inputs, and updates the calculated risk to each farm on a weekly basis. This provides visual output of risks as heat-maps and ranked lists of farms to prioritise for surveillance.

\section{MODEL OUTLINE}

\subsection{Overview}

Our aim was to produce a model that could produce relevant insights into seeking, detecting, and managing plant pests and pathogens during an incursion response. The model presented uses the example of fungal pathogens of banana crops in north Queensland, while remaining adaptable to other areas and threatening organisms, to act as an exploratory tool for incursion response managers in a variety of conceivable settings.

The model was adapted from that of Baxter et al. (2015) to incorporate multiple risk pathways, inputs of tracing and surveillance data, and sequential updating of risk estimates based on actual or simulated surveillance results. The approach taken was broadly similar to the surveillance-focussed analysis of Potts et al. (2013), with substantive differences including the incorporation of treatment of known infected farms, multiple quarantine and surveillance options, and the option of replacing simulated surveillance with actual surveillance history in updating risk estimates.

\subsection{Local dynamics}

To reflect the spatio-temporal scale of management decisions during an incursion response, we modelled disease intensity at the individual farm level using weekly time-steps. We simulated pathogen infection levels as "fungal load", a composite of extent and severity equivalent to the intensity of infection per hectare. We use an inoculum size of 1 unit for simulation of an unknown incursion, and 10 for user-entered infections of pre-existing incursions. We assume that fungal load in an infected farm grows according to a logistic growth model (van der Plank 1963) up to a fixed capacity proportional to the farm's area, subject to adequate moisture (i.e. proportionally less growth in drier weeks). Fungal growth allows propagules to become available for possible spread between farms by multiple human or environmental pathways, with spread risk profiles controlled by the user (see next section). As establishment of infection propagules may depend on propagule pressure, the user can set a threshold value, at which there is 0.9 probability of establishment.

\subsection{Spread}

The risk networks within the model assume that spread risks from a source farm will decay with distance; "distance" can be measured directly, or along physical pathways. We used general exponential distancedecay functions for risk of spread from infected farm $i$ to farms $j$ connected by spread-mode $x$ : 


$$
R_{i j, x}=m_{x} \exp \left(-\alpha_{x} d_{i j, x}\right)
$$

The connection distance $d_{i j, x}$ from farm $i$ to $j$ is measured appropriately for spread-mode $x$ (see below). Users can control risk curves by entering a value for maximum risk (at "zero" distance), $m_{x}$, and the connection distance $d_{1 / 2, x}$ at which that risk is halved (so that $\alpha_{x}=\ln (2) / d_{1 / 2, x}$ above, yielding a risk of $m_{x} / 2$ ).

(a)

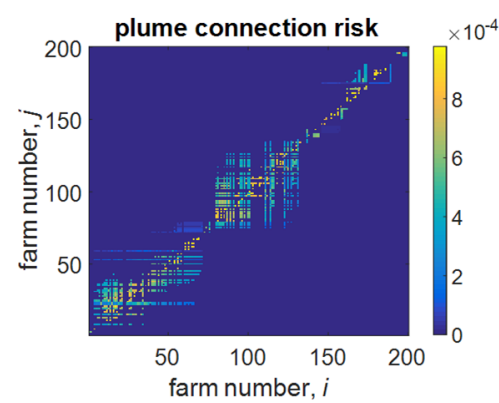

(d)

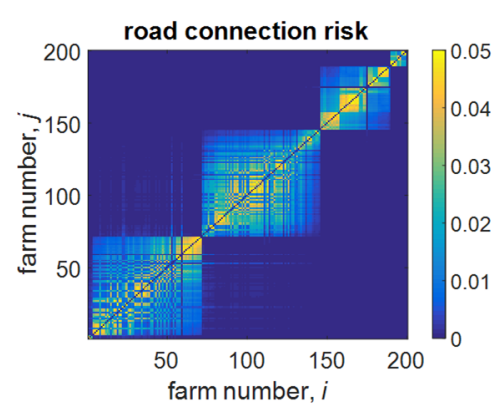

(b)

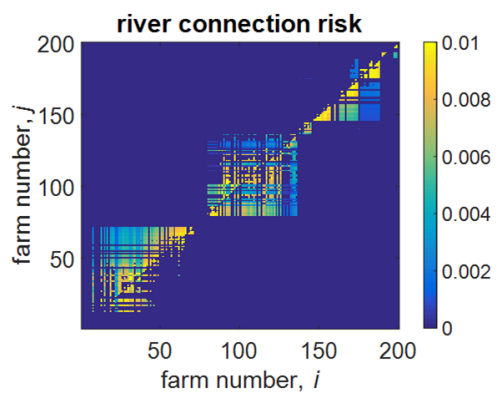

(e)

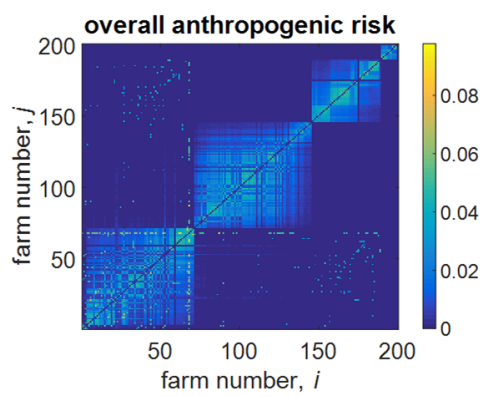

(c)

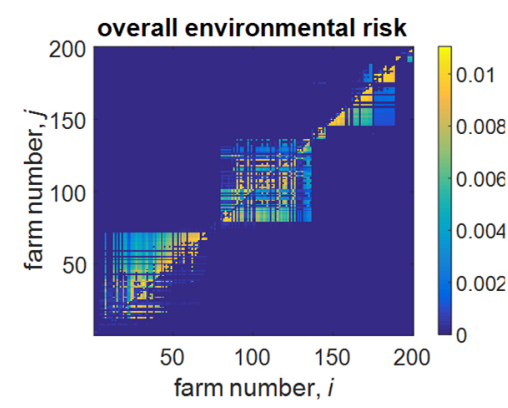

(f)

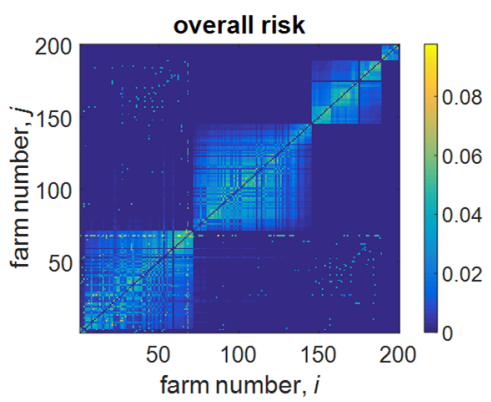

Figure 1. Spread risks by environmental (a-c) and human (d,e) transfer modes, for illustrative risk magnitudes. The colour at cell $(x=i, y=j)$ reflects risk of spread from farm $i$ to farm $j$, with yellower (brighter) colours representing higher risk. Farms are ordered by location, revealing the spatial structure in the risk networks. Risks not shown individually here include low-level background environmental and social risks, and extra initial risks derived from tracing data, whose signal can be seen in the combined anthropogenic (e) and overall risk (f), as the extra risk connections to and from farm \#68, which was the initial infested property in this example.

Connection distances are measured as Euclidean distance between farm centroids (e.g. for plume, neighbourproximity and low-level background environmental risks), or measured to and along connection routes (roads, rivers). Plume connections are unidirectional links to farms within a user-specified compass direction $\pm 30^{\circ}$. River connections are also unidirectional and are allowed between farms adjacent to major rivers flowing to the farm with the lower elevation centroid. Any farms within $5 \mathrm{~km}$ of a river were considered adjacent to allow for tributaries and overland flow in wetter conditions (without modelling water flow itself). The neighbour-proximity risk calculation fixes $m=1$, and sets $\alpha$ by assigning a user-entered maximum neighbour risk (any value between 0 and 1) to the lowest between-neighbour distance in the network. A lowlevel background socio-economic risk network disregards distance and is constructed as a symmetric risk matrix with randomly generated connection probabilities up to a user-entered maximum value.

\subsection{Detection and intervention}

We modelled probabilistic detection of the pathogen as increasing with infection intensity, $N$, at a farm, as

$$
\mathrm{P}_{\text {method }}(\text { detect })=1-\exp \left(-\delta_{\text {method }} N\right) \text {. }
$$

where "method" denotes either passive (casual) or active surveillance. Users can control the steepness of the detection curve by entering the probability of detection at the inoculum quantity, $N=1$. Following detection, treatment reduces a farm's fungal load capacity by $99 \%$, to reflect destruction of host plants while allowing persistence of fungal spores in the soil (Ploetz 2006). 
The model selects sites based on their estimated risk to simulate active surveillance, subject to a limited searchable area per week. In this paper we illustrate straightforward risk-ranked surveillance prioritisation, but a risk-weighted random site-selection algorithm (Parnell et al. 2014) is also available.

We modelled four options for quarantine restrictions (Table 1), which reduce anthropogenic connections, with an optional "leakage" rate allowing for imperfect control of movements.

Table 1. Quarantine approaches and their implementation

\begin{tabular}{|l|l|}
\hline Blanket: & Quarantine restrictions applied uniformly across the entire region, with leakage rate as entered by user. \\
\hline Target: & Known infested properties isolated with $100 \%$ efficacy, doubling the entered leakage rate elsewhere. \\
\hline Path-based: & As Blanket, with risk reduced to double the distance-by-road used to calculate risk. \\
\hline Radial link-based: & As Path-based, with all connections within the median distance of known-infested farms reduced. \\
\hline
\end{tabular}

\subsection{Incorporating data from tracing and surveillance operations}

The estimated risk to each farm can also incorporate initial risks from "traced" data, which is obtained during a response program by identifying connections between farms of interest. Tracing data files (in simple CSV format) provide necessary information on identity of farm pairs with traced connections, mode(s) of connection between each pair of farms, and estimated risk from those connections.

We also allow users to upload surveillance history into the software, which overrides simulated surveillance to give response-relevant updated risk estimates. Surveillance history data files (also in CSV format) require columns containing survey week, farm identity, actively surveyed or not (allowing for uploading of passive detections), and whether the pathogen was detected.

To incorporate surveillance results (whether user-entered or model-simulated) into estimated risk calculations, we used Bayesian updating with a presence/absence approach, which is applicable to decisions made in the course of an incursion response. If $v_{i}(t)$ is the estimated risk at time $t$ of farm $i$ being infected, but no infection was detected, we have:

$$
\begin{aligned}
& \mathrm{P}(\text { undetected } \mid \text { infected })=\left(1-p_{\text {passive }, i}\right)\left(1-\text { looked }_{i} p_{\text {active }, i}\right)= \\
& \mathrm{P}(\text { undetected } \mid \text { uninfected })=1 \\
& \mathrm{P}(\text { infected } \mid \text { missed })
\end{aligned}
$$

where the detection probabilities $p_{\text {method }}$ are again scaled to the inoculum amount, i.e. detection of a fungal

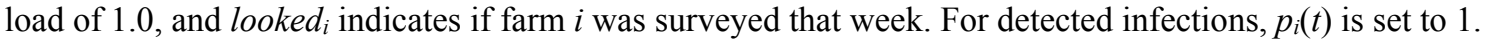
The next week's estimated risk of being infected is found by combining $p_{i}(t)$ with this week's spread risk:

$$
v_{i}(t+1)=1-\left(1-p_{i}(t)\right)\left(1-\Sigma_{j} R_{i j, x}(t) v_{j}(t)\right) .
$$

\subsection{Spatiotemporal Visualisation}

To allow for a richer user experience a web interface to the model was developed. The interface allows users to run the model under many risk/response scenarios, visualise risk and farm surveillance priority and save results. The interface is built using the ESRI ArcGIS online platform and could be accessed using any modern web browser. Users can interact with the interface as follows:

1. The user is presented with an entry screen where they can either model a new instance or retrieve the results of a previously modelled instance;

2. The user is presented with a basemap consisting of a satellite mosaic from multiple sources (including Landsat, DigitalEarth and RapidEye);

3. The user is also presented with spatial layers representing banana farms, roads and rivers. The spatial layer files were downloaded from the Queensland Government repository (QSpatial), and clipped to a regional area of interest. The user can choose to make some, all or none of the layers visible;

4. The user can specify model parameters and whether to include road and river networks in the model;

5. The user can choose to run the model with the given parameters;

6. After the model has executed, the user is presented with a visualisation consisting of a heat map and ranked list of surveillance priority. The heat map represents the mean risk-of-infection estimates from multiple simulation model runs, with higher risk farms coloured in 'warm' colours (such as red or orange) and low risk farms coloured in 'cold' colours (such as blue or green). No colour is assigned to farm with zero risk. The surveillance priority consists of a ranked list of risk (not including known infections) as 
well as markers placed over the highest-risk farms. The visualisation also contains a 'slider', allowing the user to see how the risk and ranked surveillance priority changes over time;

7. After analysing the results, the user can either save the results and parameters to PDF files or can execute another simulation.

\section{RESULTS}

\subsection{Model dynamics}

The illustrative results shown here for an uncontrolled incursion reflect the logistic growth within farms leading to the infection's spread and growth (Fig 2a). In the example shown, infection has reached every farm within six years, reaching region-wide capacity within a decade.

(a)

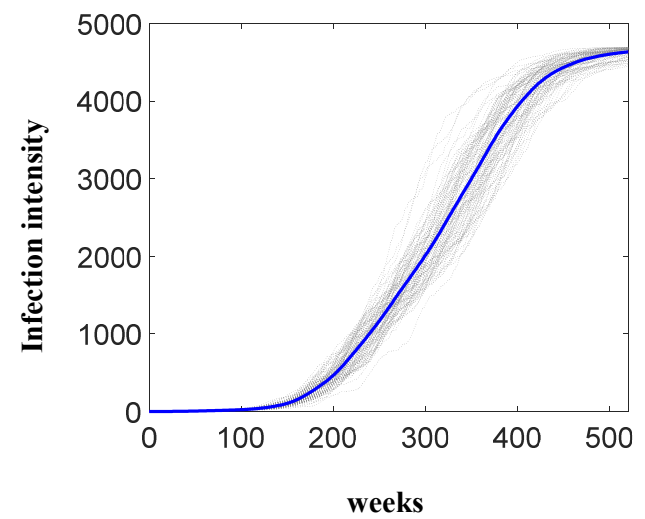

(b) Managed (radial link-based quarantine)

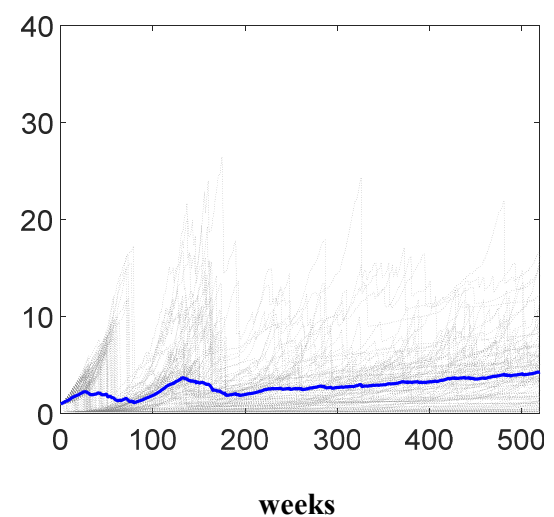

Figure 2. Example of model trajectories for an uncontrolled outbreak and one detected and managed using radial-link based quarantine. Mean infection intensity is shown in blue along with 100 indicative runs (thin gray dotted-lines). The sharp drops in Fig 2(b) are caused by detection and treatment of the disease. The quarantine leakage rate of 0.01 , coupled with residual fungal presence after treatment of infected farms, means that the infection inevitably spreads but is delayed considerably (runs of up to 100 years, not shown, indicate slow approach to a fungal load of approximately 47 , i.e. $1 \%$ of overall capacity so that all farms are eventually infected, detected and treated). Other quarantine methods (cf. Tables 1,2) show similar behaviour.

Management interventions following (simulated, non-immediate) detection show relative success of controlling the outbreak, by treating infected properties when detected and imposing quarantine restrictions (Table 2). The overall infection load (in ha-weeks of infection) and impact to individual farms (measured as site-weeks of infection) is reduced as spread of the pathogen is suppressed. Radial link-based (Fig 2b) and path-based quarantine are the best-performing measures. Interestingly, these strategies strike a balance between local focus and more broad-scale interventions, outperforming both overly-focussed "targeted" quarantine and the region-wide blanket restrictions.

Table 2. Expected impacts summed over 520 weeks (mean of 1,000 simulations), providing an indication of total impact of the incursion and the effectiveness of each quarantine option

\begin{tabular}{|l|c|c|c|c|c|}
\hline Quarantine strategy: & Unmanaged & blanket & targeted & path-based & link-based \\
\hline Total infection load (ha-weeks) & $1,041,993$ & 2,889 & 2,918 & 1,773 & 1,752 \\
\hline Total site infections (farm-wks) & 70,396 & 13,408 & 11,606 & 7,058 & 6,795 \\
\hline
\end{tabular}

\subsection{Incorporating data during incursion response}

Figure 3 illustrates example use of the BIOSPARK software, showing the user interface (Fig 3a) and output under different scenarios (Fig 3b,c). Inclusion of tracing data (Fig 3b) may identify previously overlooked high-risk sites and flag them for surveillance (e.g. farm 45, towards bottom-left). Fig 3(c) further incorporates 
user-uploaded surveillance history data and re-estimates risk: in this case the user has reported visiting the farm \#45 from weeks 6-10 without detection, and the farm has been de-prioritised in risk rankings.

(a)

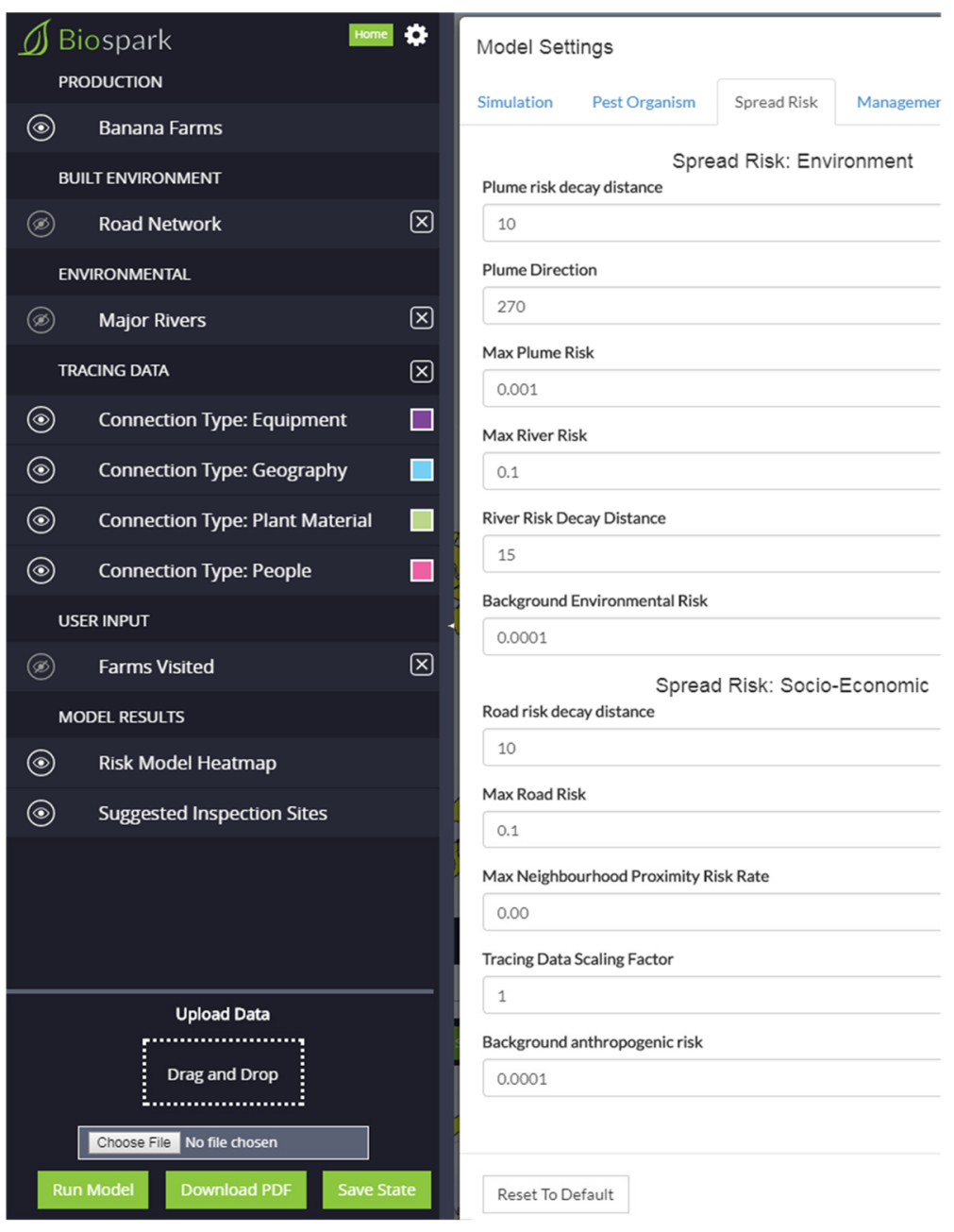

(b)

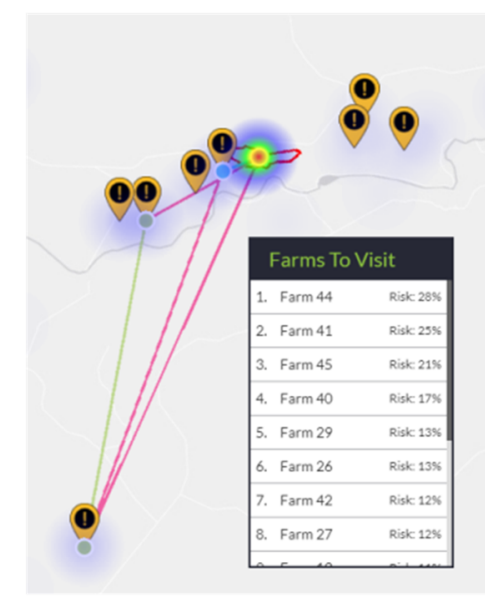

(c)

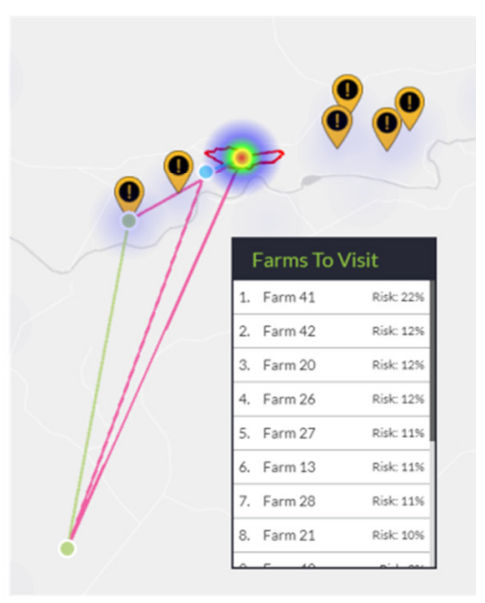

Figure 3. Illustrative examples of BIOSPARK user interface (a) and output (b,c). The user menus (a) allow control of visualisation, upload of data, and parameter settings. In this example the heat maps (b,c; detail only) depict risks to farms in the $11^{\text {th }}$ week of an incursion detected at the site outlined in red, and indicate highly-ranked farms for surveillance consideration with orange flags and a pop-up table. Inclusion of traced risks (b; coloured by type) affects the surveillance ranking of farms, highlighting a cross-river risk previously overlooked. Similarly, inclusion of past surveillance data can affect the surveillance ranking (c). Note that while the spatial layout of farms is derived from QSpatial, the infested property and risks to other sites depicted here are for example purposes only.

\section{DISCUSSION AND CONCLUSIONS}

We have produced a model that simulates a fungal pathogen spreading in a plausible manner, and following detection and treatment of the disease, the successful arrest of its spread through quarantine restrictions. Quarantine approaches that exploit the location of strong links in the risk networks can perform better than either broad-scale restrictions or those that overly focus on the known infected properties.

The model provides the basis for the BIOSPARK visualisation tool, which allows managers to explore possible scenarios and update their decisions with data when available. We have endeavoured to include diverse and important spread vectors but to include options for all possible vectors would be prohibitively complicated and confusing for the user. However, the existing options have the potential for broader interpretation to reflect bespoke needs. For example, users could re-parameterise background omnidirectional environmental 
risk (in which risk decays radially over Euclidean distance), and, with due caution, interpret the result in terms of spread risk by animal vectors such as birds or bats.

Future developments would ideally include considerations of different organisms, flexibility in number of risk networks to be entered, and application of the model to different jurisdictions.

\section{ACKNOWLEDGEMENTS}

We thank the Visualisation and eResearch (ViseR) team at QUT for software interface design and development. We thank the reference group for project PBCRC-2100 and participants in BIOSPARK workshops for valuable feedback and comments. We acknowledge the support of the Australian Government's Cooperative Research Centres Program.

\section{REFERENCES}

Barkley, P., T. Schubert, G. Schutte, K. Godfrey, V. Hattingh, G. Telford, G. Beattie, and K. Hoffman (2014). Invasive pathogens in plant biosecurity. Case study: citrus biosecurity. Pages 547-592 in F. Gordh and S. McKirdy, editors. Handbook of Plant Biosecurity. Springer, Dordrecht, Netherlands.

Baxter, P.W.J., S. Parnell, and G. Hamilton (2015). Quarantine and surveillance strategies for plant pathogen detection and control. Pages 1261-1267 in T. Weber, M.J. McPhee, and R.S. Anderssen, editors. MODSIM2015, 21st International Congress on Modelling and Simulation. Modelling and Simulation Society of Australia and New Zealand, Gold Coast, Australia.

Baxter, P.W.J., and H.P. Possingham (2011). Optimizing search strategies for invasive pests: learn before you leap. Journal of Applied Ecology 48, 86-95.

Cook, D.C., S. Liu, J. Edwards, O.N. Villalta, J.-P. Aurambout, D.J. Kriticos, A. Drenth, and P.J. De Barro (2013). Predicted economic impact of black Sigatoka on the Australian banana industry. Crop Protection $51,48-56$.

Cook, D.C., A.S. Taylor, R.A. Meldrum, and A. Drenth (2015). Potential economic impact of Panama Disease (Tropical Race 4) on the Australian banana industry. Journal of Plant Diseases and Protection 122, 229-237.

Murray, G.M., and J.P. Brennan (2010). Estimating disease losses to the Australian barley industry. Australasian Plant Pathology 39, 85-96.

Parnell, S., T.R. Gottwald, T. Riley, and F. van den Bosch (2014). A generic risk-based surveying method for invading plant pathogens. Ecological Applications 24, 779-790.

Pimentel, D., L. Lach, R. Zuniga, and D. Morrison (2000). Environmental and economic costs of nonindigenous species in the United States. Bioscience 50, 53-65.

Ploetz, R.C. (2006). Fusarium wilt of banana is caused by several pathogens referred to as Fusarium oxysporum f. sp cubense. Phytopathology 96(6), 653-656.

Potts, J.M., M.J. Cox, P. Barkley, R. Christian, G. Telford, and M.A. Burgman (2013). Model-based search strategies for plant diseases: a case study using citrus canker (Xanthomonas citri). Diversity and Distributions 19, 590-602.

Rasmussen, R., and G. Hamilton (2012). An approximate Bayesian computation approach for estimating the parameters of complex environmental processes in a cellular automata. Environmental Modelling and Software 29, 1-10.

Simberloff, D (2003). Eradication-preventing invasions at the outset. Weed Science 51, 247-253.

van der Plank, J.E. (1963). Plant Diseases: Epidemics and Control. New York, NY, USA, Academic Press.

Westphal, M.I., M. Browne, K. MacKinnon, and I. Noble (2008). The link between international trade and the global distribution of invasive alien species. Biological Invasions 10, 391-398. 